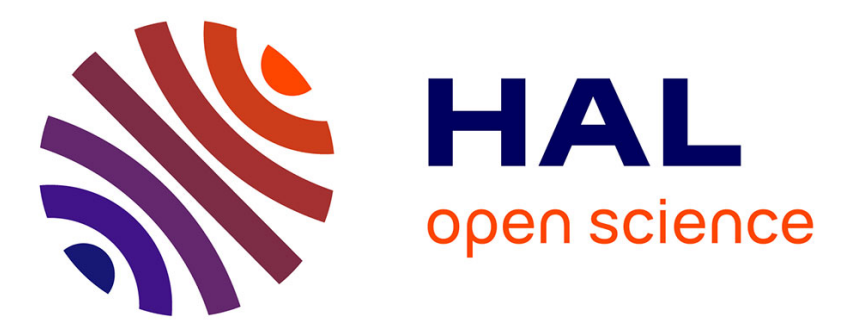

\title{
Robust sensor localization with locally-computed, global Hinf-design
}

\author{
Anton Korniienko, Usman A Khan, Gérard Scorletti
}

\section{To cite this version:}

Anton Korniienko, Usman A Khan, Gérard Scorletti. Robust sensor localization with locallycomputed, global Hinf-design. 2016 IEEE ACC, Jul 2016, Boston, MA, United States. pp.6375 6380, 10.1109/ACC.2016.7526672 . hal-01484252

\section{HAL Id: hal-01484252 \\ https://hal.science/hal-01484252}

Submitted on 7 Mar 2017

HAL is a multi-disciplinary open access archive for the deposit and dissemination of scientific research documents, whether they are published or not. The documents may come from teaching and research institutions in France or abroad, or from public or private research centers.
L'archive ouverte pluridisciplinaire HAL, est destinée au dépôt et à la diffusion de documents scientifiques de niveau recherche, publiés ou non, émanant des établissements d'enseignement et de recherche français ou étrangers, des laboratoires publics ou privés. 


\title{
Robust sensor localization with locally-computed, global $H_{\infty}$-design
}

\author{
Anton Korniienko, Usman A. Khan, and Gerard Scorletti
}

\begin{abstract}
This paper considers sensor localization in $\mathbb{R}^{m}$, i.e. the problem to find the positions of an arbitrary number of sensor nodes in the presence of at least $m+1$ anchor nodes, given only the inter-node distances. Assuming that the sensors lie in the convex hull of the anchors, we provide a linear, continuous-time update at each sensor that uses barycentric coordinates and Cayley-Menger determinants. In this paper, we consider design enhancements by adding a dynamic controller in the feed-forward loop of the location estimator at each sensor. We show that the dynamic controller has the ability to both speed-up the convergence and improve the transient behavior, while ensuring a certain disturbance rejection that could be introduced by inter-node communication. The design of the local controllers is based on the input-output approach, $H_{\infty}$ design and does not require the knowledge of global parameters. Simulations illustrates the concepts described in the paper.
\end{abstract}

\section{Index Terms}

Sensor localization, Robust control, Decentralized control, Control of networks, Sensor fusion.

\section{INTRODUCTION}

This paper considers sensor localization where the problem is to find the positions of sensors placed in a Euclidean space, $\mathbb{R}^{m}$, given a certain number of anchors with perfectly known locations. It is well-known that the localization problem requires at least $m+1$ anchors, in general, given only pairwise distances. However, problems with a large number of sensors typically require a much higher number of anchors. The literature on localization is largely based on the well-known triangulation and trilateration principles, see [1] for details, or in some cases, a combination of both. Centralized localization algorithms include: maximum likelihood estimators, [2]; multidimensional scaling (MDS), [3], [4]; and optimization-based methods, see [5]; for additional work, see [6], [7].

On the other hand, a significant attention is also devoted to distributed localization algorithms that can be characterized into two classes: multilateration and successive refinements. In multilateration algorithms, [8], [9], each sensor estimates its distance from the anchors, possibly via multi-hop communication, and then calculates its location via trilateration. Successive refinement algorithms utilize an iterative optimization of a cost function and can be found in, e.g., [10]. Reference [11] discusses a Self-Positioning Algorithm (SPA) that provides a GPS-free positioning and builds a relative coordinate system. Other related work also consists of graph-theoretic approaches [12], [13], and probabilistic methods, [14], [15].

AK and GS are with Laboratoire Ampère, Université de Lyon, École Centrale de Lyon, 69134 Ecully Cedex, France, ffirst name $\}$. last name $\}$ dec-lyon.fr. Their work is supported by a grant from la Région Rhône-Alpes.

UAK is with the Department of Electrical and Computer Engineering at Tufts University, Medford, MA 02155, USA, khan@ece.tufts.edu. His work is supported by an NSF Career award \# CCF-1350264. 
Of significant relevance to this paper are Refs. [1], [16], where a discrete-time algorithm, named DILOC, is described assuming that the sensors lie inside the convex hull of at least $m+1$ anchors in $\mathbb{R}^{m}$. DILOC is completely local and distributed: each sensor finds a triangulation set of $m+1$ neighboring nodes (sensors and/or anchors) such that the sensor in question lies inside the convex hull of this set. The locations estimates are subsequently updated as a linear combination of the neighboring locations (estimates) in the triangulation set. The coefficients in this linear update are the barycentric coordinates, attributed to August F. Möbius, [17]. Assuming that a triangulation set exists at each sensor, DILOC converges to the true sensor locations. It is important to note that DILOC is linear and only requires $m+1$ anchors regardless of the number of sensors as long as the deployment (convexity) conditions are satisfied.

In [18], we provide DILOC-CT that is a continuous-time analog of DILOC and show that by using a proportional controller in the location estimators, the convergence speed can be increased arbitrarily. Since this increase may come at the price of unwanted transients especially when there is disturbance introduced by the network, we consider dynamic controllers that guarantee certain performance and disturbance rejection properties. However, the controller design in [18] is not local, i.e., it requires all of the barycentric coordinates to be known in order to compute the controller parameters. In this paper, we describe a distributed control design that is completely local and only relies on a spectral bound of the matrix of barycentric coordinates. The design in this paper, thus, can be directly implemented at the sensors and no central computation is required. Our approach is based on $H_{\infty}$ design principles and uses the input-output approach, see e.g., [19]-[21].

We now describe the rest of the paper. Section II describes the problem and recaps DILOC-CT while Section III presents the interpretation of the problem under consideration in frequency domain ( $s$-domain). Section IV discusses the main results on using the input-output approach for the decentralized controller design. Section V illustrates the concepts and Section VI concludes the paper.

Notation: The superscript, ' $T$ ', denotes a real matrix transpose while the superscript, ' $*$ ', denotes the complexconjugate transpose. The $N \times N$ identity matrix is denoted by $I_{N}$ and the $n \times m$ zero matrix is denoted by $0_{n \times m}$. The dimension of the identity or zero matrix is omitted when it is clear from the context. The diagonal aggregation of two matrices $A$ and $B$ is denoted by $\operatorname{diag}(A, B)$. The Kronecker product, denoted by $\otimes$, between two matrices, $A$ and $B$, is defined as $A \otimes B=\left[a_{i j} B\right]$. We use $T_{x \rightarrow y}(s)$ to denote the transfer function between an input, $x(t)$, and an output, $y(t)$. With matrix, $G$, partitioned into four blocks, $G_{11}, G_{12}, G_{21}, G_{22}, G \star K$ denotes the Redheffer product, [22], i.e., $G \star K=G_{11}+G_{12} K\left(I-G_{22} K\right)^{-1} G_{21}$. Similarly, $K \star G=G_{22}+G_{21} K\left(I-G_{11} K\right)^{-1} G_{12}$. For a stable LTI system, $G,\|G\|_{\infty}$ denotes the $H_{\infty}$ norm of $G$. For a complex matrix, $P, \bar{\sigma}(P)$ denotes its maximal singular value while $\rho(P)$ denotes its spectral radius. Finally, the symbols, ' $\geq$ ' and '>, denote positive semi-definiteness and positive-definiteness of a matrix, respectively.

\section{Preliminaries And Problem Formulation}

In this paper we consider the problem of Distributed Localization problem in continuous time (DILOC-CT) see [1], [16], [18], [23] for details. It consists in a network of $M$ sensors, in the set $\Omega$, with unknown locations, 
and $N$ anchors, in the set $\kappa$, with known locations, all located in $\mathbb{R}^{m}, m \geq 1$; let $\Theta=\Omega \cup \kappa$. Let $\mathbf{x}_{i}^{*} \in \mathbb{R}^{1 \times m}$ denote the true location of the $i$ th sensor, $i \in \Omega$; similarly, $\mathbf{u}_{j} \in \mathbb{R}^{1 \times m}, j \in \kappa$, for an anchor. We assume that each sensor knows its distances to the nearby nodes (sensors and/or anchors). The problem is to find the locations of the sensors in $\Omega$. For this purpose, let $\mathbf{x}_{i}(t) \in \mathbb{R}^{1 \times m}$ denote the $m$-dimensional row-vector of sensor $i$ 's location estimate, where $t \geq 0$ is the continuous-time variable. DILOC-CT is given by the following equation, where $(t)$ is dropped in the sequel for convenience:

$$
\dot{\mathbf{x}}_{i}=\alpha\left(-\mathbf{x}_{i}+\mathbf{r}_{i}\right) ; \mathbf{r}_{i} \triangleq \sum_{j \in \Theta_{i} \cap \Omega} p_{i j} \mathbf{x}_{j}+\sum_{j \in \Theta_{i} \cap \kappa} b_{i j} \mathbf{u}_{j}
$$

where $p_{i j}$ 's are the sensor-to-sensor and $b_{i j}$ 's are sensor-to-anchor barycentric coordinates and $\Theta_{i}$ is the triangulation set at sensor $i$. Note that $\Theta_{i}$ may not contain any anchor and all barycentric coordinates are positive and sum to 1 .

Let $\mathbf{x}(t) \in \mathbb{R}^{M \times m}$ (and $\mathbf{u}(t) \in \mathbb{R}^{(m+1) \times m}$ ) denote the matrix regrouping the vectors of sensor (and anchor) locations, and let $P \triangleq\left\{p_{i j}\right\}$ and $B \triangleq\left\{b_{i j}\right\}$. Since $\rho(P)<1$, it can be shown that the resulting dynamics, $\dot{\mathbf{x}}=-\alpha(I-P) \mathbf{x}+\alpha B \mathbf{u} \triangleq P_{\alpha} \mathbf{x}+B_{\alpha} \mathbf{u}, \forall \alpha>0$ converge to the true sensor locations:

$$
\lim _{t \rightarrow \infty} \mathbf{x}(t)=(I-P)^{-1} B \mathbf{u}=\mathbf{x}^{*}
$$

\section{A. Problem formulation}

Towards a more practical system, we consider the received signal at each sensor to incur a zero-mean additive disturbance, $\mathbf{n}_{i}(t)$, whose frequency spectrum lies in the interval, $\left[\omega_{z}^{-}, \omega_{z}^{+}\right]$. With this disturbance, the location estimator is given by

$$
\dot{\mathbf{x}}_{i}=\alpha\left(-\mathbf{x}_{i}+\mathbf{r}_{i}+\mathbf{n}_{i}\right)
$$

We replace the proportional gain, $\alpha$, with a dynamic controller, $K(s)$; see Fig. 1 leading to the dynamical system, $T_{s}$, at each sensor. We note that the above localization algorithm is linear and in continuous-time, which allow us to use frequency-based design approach, which has advantages in handling multi-objective specifications and in treating the disturbance, usually present in High-Frequency (HF) range. The control design problem is to ensure global stability and global performance expressed in terms of a certain transient behavior, convergence rate, and disturbance rejection, all at the same time. In this paper, we relax the knowledge of $P$ and $B$, required for controller design in [18]. In particular, we propose a controller design not for a given topology, $P, B$, but for a set of all topologies bounded in the sense of maximum singular value.

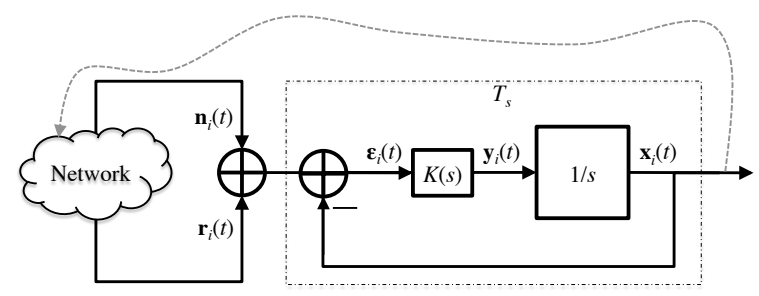

Fig. 1. DILOC-CT architecture, see [18]. 


\section{DILOC-CT WITH DyNAMIC CONTROLleR: $s$-DOMAIN ANALYSIS}

Since DILOC-CT is decoupled in the coordinates, each local system, $T_{s}$, see Fig. 1 , can be analyzed per coordinate and the same analysis can be extended to other coordinates. Recall that the network location estimate, $\mathbf{x}(t)$, is an $M \times m$ matrix where each column is associated to a location coordinate in $\mathbb{R}^{m}$. We let $x$ to be an arbitrarily chosen column of $\mathbf{x}$ corresponding to one chosen dimension. Similarly, we let $n, r, \varepsilon$, and $y$ (signals in Fig. 1) to represent $M$-dimensional vectors; for any of such vectors, the subscript $i$ denotes the chosen coordinate at sensor $i$. Similar to [18], the global system under consideration is described by:

$$
\begin{gathered}
x=\left(I_{M} \otimes T_{s}(s)\right) r^{n}, \\
{\left[\begin{array}{c}
r^{n} \\
\varepsilon^{r e f} \\
\varepsilon
\end{array}\right]=\underbrace{\left[\begin{array}{c|cc}
P & B & I_{M} \\
\hline-I_{M} & \left(I_{M}-P\right)^{-1} B & 0 \\
P-I_{M} & B & I_{M}
\end{array}\right]}_{H_{0}}\left[\begin{array}{c}
x \\
u \\
n
\end{array}\right] .}
\end{gathered}
$$

where $s$ is Laplace variable, the input vector $u \in \mathbb{R}^{N}$ represents the anchors position sent to the sensors, $n \in \mathbb{R}^{M}$ is the input vector regrouping all sensor disturbance signals $n_{i}, x^{*} \in \mathbb{R}^{M}$ and $x \in \mathbb{R}^{M}$ are the vectors regrouping the true and estimated sensor location $x_{i}^{*}$ and $x_{i}$. The signal $r^{n} \in \mathbb{R}^{M}: r^{n} \triangleq r+n=P x+B u+n$, is the vector regrouping all local sensor inputs $r_{i}^{n}$. The signal $\varepsilon^{r e f} \in \mathbb{R}^{M}: \varepsilon^{\text {ref }} \triangleq x^{*}-x=\left(I_{M}-P\right)^{-1} B u-x$ is the vector

regrouping all sensor localization tracking errors $\varepsilon_{i}^{r e f}$ and the signal $\varepsilon \in \mathbb{R}^{M}: \varepsilon \triangleq r^{n}-x$ is the vector regrouping all local sensor errors $\varepsilon_{i}$.

The local transfer function, $T_{s}$, identical at each sensor. It is complementary sensitivity function related to sensitivity transfer function $S(s)$ by

$$
T_{s}(s)=\frac{K(s)}{s+K(s)} ; S(s)=1-T_{s}(s)=\frac{s}{s+K(s)}
$$

Given the representation in Eqs. (3) and (4), we have

$$
T_{\left[\begin{array}{l}
u \\
n
\end{array}\right] \rightarrow\left[\begin{array}{c}
\varepsilon^{r e f} \\
\varepsilon
\end{array}\right]}=\left[\begin{array}{cc}
T_{u \rightarrow \varepsilon} \text { ref } & T_{n \rightarrow \varepsilon} \text { ref } \\
T_{u \rightarrow \varepsilon} & T_{n \rightarrow \varepsilon}
\end{array}\right]=\left(I_{M} \otimes T_{s}\right) \star H_{0} .
$$

Let us define the following global transfer functions:

$$
\begin{gathered}
S^{g}(s) \triangleq T_{u \rightarrow \varepsilon^{r e f}}(s), \quad T^{g}(s) \triangleq \frac{K(s)}{s} T_{n \rightarrow \varepsilon}(s), \\
K S^{g}(s) \triangleq K(s) T_{n \rightarrow \varepsilon}(s) .
\end{gathered}
$$

In [18], we proposed a method for control design, $K(s)$, that ensures the global stability and performance expressed in terms of frequency-dependent constraints on transfer functions, $S^{g}, T^{g}$ and $K S^{g}$. However, as pointed out before, the control law could be designed only if the interconnection topology, i.e., $P$ and $B$, is known in advance. In this paper, we propose an extension of the method in [18] to a set of possible interconnection topologies defined in terms of maximum singular value on interconnection matrix, $P$ :

$$
\bar{\sigma}(P) \leq \gamma<1
$$


Clearly, the convergence requirement for DILOC-CT, i.e., $\rho(P)<1$, is implied by the above inequality. However, the conditions for the existence of a dynamic controller that ensures the global stability and performance for all interconnections satisfying Eq. (8) are potentially more restrictive that only for one given interconnection, $P$. For this reason, we propose a reduction that is justified by the following Theorem.

Theorem 1: Consider DILOC-CT described in Fig. 1. If the local dynamic controller, $K(s)$, (i) ensures the global system stability and (ii) does not contain pure derivative actions, i.e., $K(0) \neq 0$, then it ensures global tracking performance, i.e., DILOC-CT converges to the true sensor locations, $x^{*}$ :

$$
\lim _{t \rightarrow \infty} x(t)=(I-P)^{-1} B u=x^{*} .
$$

Proof: See [23] for the proof.

Based on Theorem 1, and the fact that the speed of convergence is proportional to the static gain of controller, see [18], we can reduce the global performance transfer function by eliminating the inputs and outputs related to the tracking performance: $u$ and $\varepsilon^{r e f}$, in Eqs. (4) and (6). We thus obtain the following reduced global system description:

$$
T_{n \rightarrow \varepsilon}=\left(I_{M} \otimes T_{s}\right) \star H_{1},
$$

where ' $\star$ ' is the Redheffer product, with $T_{s}$ defined by Eq. (5) and $H_{1}$ by

$$
H_{1}=\left[\begin{array}{c|c}
P & I \\
\hline P-I & I
\end{array}\right] .
$$

Hence, the following control design problem can formulated:

Problem 1 (Control problem): Given the global system in Eq. (10), for all possible sensor interconnection topology such that, P satisfies Eq. (8), find the local controller, $K(s)$, such that the global system is stable and satisfies the following frequency constraints:

$$
\begin{array}{cl}
|K(j \omega)| \geq \Omega_{K}(\omega), & \text { in LF range, } \\
\bar{\sigma}\left(T^{g}(j \omega)\right) \leq \Omega_{T}(\omega), & \text { in HF range, } \\
\bar{\sigma}\left(K S^{g}(j \omega)\right) \leq \Omega_{K S}(\omega), & \text { in HF range. }
\end{array}
$$

Furthermore, each sensor should be able to solve the problem locally, i.e., the computation of $K$ is to be performed at each sensor.

We now briefly explain the frequency constraints. The first constraint, $\Omega_{K}$, ensures zero steady-state error $(K(0) \neq$ 0 ) and provides a handle on the speed of convergence for each sensor to its true location. The second and third constraints, $\Omega_{T}$ and $\Omega_{K S}$, impose a maximum bandwidth on $T^{g}$ and a maximum gain on $K S^{g}$, which, in turn, limits the disturbance amplification by each sensor in high-frequency range. Specifics on these constraints are tabulated in Table I and are further elaborated in Section V.

It is important to note, that the proposed result is general enough to cover the case of not reduced problem, i.e., imposing the frequency constraints for all transfer functions, $S^{g}, K S^{g}$ and $T^{g}$, as it is proposed in [18]. However, 
TABLE I

DILOC-CT DESIGN SPECIFICATIONS

\begin{tabular}{|c|c|c|}
\hline Performance specs. & Frequency constraint & Range \\
\hline \hline Zero steady-state & \multirow{2}{*}{ Global stability and $|K(j \omega)| \geq K_{0}$} & \multirow{2}{*}{ LF } \\
\cline { 1 - 2 } Conv. speed & $-60 \mathrm{~dB} / \mathrm{dec}$ slope for $\bar{\sigma}\left(T^{g}(j \omega)\right)$ & \multirow{2}{*}{ HF } \\
\cline { 1 - 2 } Disturbance reduction & $-40 \mathrm{~dB} / \mathrm{dec}$ slope for $\bar{\sigma}\left(K S^{g}(j \omega)\right)$ & \\
\cline { 2 - 3 } &
\end{tabular}

to reduce the computational load such that the proposed design method can by easily implemented at each sensor, the reduced version of the Problem 1 is solved in section V.

\section{Main Results}

We now describe the main results of this paper. Before we start, in subsection IV-A, we recap the input-output approach and present its main Theorem: Theorem of graph separation which can be used to solve the problem under consideration for one given interconnection topology. Then, in subsection IV-B, we extend the result to a more general case where the interconnection topology is not fixed but supposed to belong to a bounded set. Finally, in subsection IV-C, we specialize the obtained result to the case of sensor localization under consideration.

\section{A. Input-output Approach}

We now describe the input-output approach used to solve Problem 1. We use the concept of dissipativity taken from [19]-[21], [24], a simplified version of which is defined below.

Definition 1 (Dissipativity): An LTI, stable, and causal operator, $H$, is strictly $\{X, Y, Z\}$-dissipative, where $X=$ $X^{T}, Y, Z=Z^{T}$, are real matrices such that

$$
\left[\begin{array}{cc}
X & Y \\
Y^{T} & Z
\end{array}\right]
$$

is full-rank; if $\exists \varepsilon>0$ such that for almost all $\omega>0$

$$
\left[\begin{array}{c}
I \\
H(j \omega)
\end{array}\right]^{*}\left[\begin{array}{cc}
X & Y \\
Y^{T} & Z
\end{array}\right]\left[\begin{array}{c}
I \\
H(j \omega)
\end{array}\right] \leq-\varepsilon I .
$$

If the inequality in Eq. (12) is satisfied with $\varepsilon=0$, the operator is said to be $\{X, Y, Z\}$-dissipative.

Based on the dissipativity characterization of two interconnected systems, $\mathcal{T}$ and $\mathcal{H}$, the following result could be obtained ensuring the stability and performance of the interconnection $\mathcal{T} \star \mathcal{H}$.

Theorem 2: Given $\eta>0$, a stable LTI system, $\mathcal{H}$, interconnected with an LTI system, $\mathcal{T}$, and real matrices, $\mathcal{X}=$ $\mathcal{X}^{T} \geq 0, \mathcal{Y}, \mathcal{Z}=\mathcal{Z}^{T} \leq 0$ of appropriate dimensions, if

(i) $\mathcal{H}$ is $\left\{\operatorname{diag}\left(\mathcal{X},-\eta^{2} I\right), \operatorname{diag}(\mathcal{Y}, 0), \operatorname{diag}(\mathcal{Z}, I)\right\}$-dissipative, and

(ii) $\mathcal{T}$ is strictly $\left\{-\mathcal{Z},-\mathcal{Y}^{T},-\mathcal{X}\right\}$-dissipative,

then the global system, $\mathcal{T} \star \mathcal{H}$, is stable and

$$
\|\mathcal{T} \star \mathcal{H}\|_{\infty} \leq \eta .
$$


Proof: The proof of Theorem 2 can be found in [21], and it relies on a version of the graph-separation theorem used in [19] for the stability and an S-procedure, [25], for the performance. It can also be seen as a generalization of the Kalman-Yakubovich-Popov lemma, [26].

Remark 1: We note that for a given interconnection topology, $P$, Problem 1 can be solved by applying Theorem 2 with $\mathcal{H}=H_{1}, \mathcal{T}=\left(I \otimes T_{s}\right)$ and dissipativity matrices chosen as $\mathcal{X}=Q \otimes X, \mathcal{Y}=Q \otimes Y, \mathcal{Z}=Q \otimes Z$ with a positive-definite symmetric matrix, $Q \in \mathbb{R}^{M \times M}$, see [18] for more details. However, for this purpose, the knowledge of interconnection matrix, $H_{1}$ (and thus $P$ ), is necessary. In turn, the knowledge of all barycentric coordinates, $P$, to compute the local controller may be restrictive from an application point of view. Indeed, if the barycentric coordinates are known in advance, the position of each sensor could be easily computed at some central computer and then transmitted to the sensors. In order to avoid such knowledge, the condition (i) of Theorem 2 should be satisfied not only for one given interconnection $P$ but for a set of all possible interconnections. In this paper, we present this extension based on the idea of representing the interconnection matrix, $P$, as an uncertainty and exploiting the fact that it is bounded in terms of its spectral radius, $\rho(P) \leq \gamma<1$, implying Eq. (8).

\section{B. Global Performance for a set of Topologies}

One approach to solve the Problem 1 could be to derive a similar constraint on the augmented interconnection matrix $H_{1}$ based on the constraint (8), see Eq. (10):

$$
\bar{\sigma}(P) \leq \gamma<1 \Rightarrow \bar{\sigma}\left(H_{1}\right) \leq \tilde{\gamma}<1
$$

Depending on the performance under consideration, the last condition could be very restrictive and actually never be applied in practice. For this reason, in this paper we propose to transform the system Eq. (10) in the form of an LFT (Linear Fractional Transform) interconnection of local subsystem dynamics, $T_{s}$, and one or several (repeated) interconnection matrices, $P$. Such a transformation is general enough to cover a large set of performance specifications. It could be easily performed in the case of sensor localization problem under consideration as shown later. In this case, it is possible to derive less restrictive global performance conditions based on the constraint in Eq. (8), instead of Eq. (14). Let us thus consider the following general description of the transformed global system:

$$
\begin{gathered}
p=\operatorname{diag}\left(I_{M} \otimes T_{s}, I_{m} \otimes \bar{P}\right)(q), \\
{\left[\begin{array}{c}
q \\
z
\end{array}\right]=\underbrace{\left[\begin{array}{ll}
H_{11} & H_{12} \\
H_{21} & H_{22}
\end{array}\right]}_{H}\left(\left[\begin{array}{l}
p \\
w
\end{array}\right]\right),}
\end{gathered}
$$

is a finite-dimensional, stable LTI system, $w(t)$ is the input vector of dimension $n_{w}, z(t)$ is the output vector of dimension $n_{z}$, and $q(t), p(t)$, are internal signals of dimensions $n_{g}$. The local subsystem is $T_{s}=G \star K$ with $n_{l}$ inputs and outputs ${ }^{1}$. We note that $n_{g}=(1+m) n_{l} \times M$. Please also note that, to cover general result, the systems

${ }^{1}$ Without loss of generality and for the ease of notation, the square local system case is presented here. 
under consideration in Eq.(15) could be Multi-Input Multi-Output (MIMO) if $n_{l}>1$. In this case, interconnection matrix $\bar{P}=P \otimes I_{n_{l}}$ and its maximum singular value respect the same condition as $P$, i.e., Eq. (8).

The global transfer function between external input, $w$, and output, $z$, is $T_{w \rightarrow z}=\operatorname{diag}\left(I_{M} \otimes T_{s}, I_{m} \otimes \bar{P}\right) \star H$, and its $H_{\infty}$-norm is ensured by the local controller, $K$, by the following theorem providing the main result of the paper.

Theorem 3: Given $\eta>0$ and $\gamma>0$, a stable LTI system, $H$, a local plant, $G$, and real matrices, $X=X^{T} \geq$ $0, Y, Z=Z^{T}$, if there exist

(i) positive-definite matrices $Q$ and $D$ of appropriate dimensions, such that $H$ is $\left\{\right.$ diag $\left(Q \otimes X,-D \otimes I,-\eta^{2} I\right)$, diag $(Q \otimes Y, 0,0), \operatorname{diag}(Q$ dissipative, and

(ii) a local controller, $K$, such that $T_{s}=G \star K$ is strictly $\left\{-Z,-Y^{T},-X\right\}$-dissipative,

then the local controller, $K$, ensures that the global system, $\left(I \otimes T_{s}, I \otimes \bar{P}\right) \star H$, is stable and

$$
\left\|\left(I \otimes T_{s}, I \otimes \bar{P}\right) \star H\right\|_{\infty} \leq \eta
$$

for all interconnection matrices $\bar{P}$ satisfying

$$
\bar{\sigma}(\bar{P}) \leq \gamma
$$

Proof: See [23] for the proof.

\section{Local Control for Global Performance of DILOC-CT}

In the previous subsection, we presented the Theorem that allows to solve Problem 1 for all interconnection topologies that satisfy Eq. (8) if the global system is transformed in the LFT form of Eq. (15). In this subsection, we show how this transformation is made for DILOC-CT application under consideration in order to ensure the frequency depended bounds, Eq. (11) by decentralized control, $K$.

Note that $H_{1}$ in Eq. (10) can be written in form of an LFT of one interconnection matrix, $P$, since:

$$
H_{1}=\left[\begin{array}{cc}
0 & I_{M} \\
-I_{M} & I_{M}
\end{array}\right]+\left[\begin{array}{c}
I_{M} \\
I_{M}
\end{array}\right] P\left[\begin{array}{ll}
I_{M} & 0
\end{array}\right] .
$$

Therefore, Eq. (10) can be equivalently written as:

$$
\begin{gathered}
{\left[\begin{array}{c}
x \\
x^{p}
\end{array}\right]=\operatorname{diag}\left(I_{M} \otimes T_{s}, P\right)\left(\left[\begin{array}{c}
r^{n} \\
r^{p}
\end{array}\right]\right),} \\
{\left[\begin{array}{c}
r^{n} \\
r^{p} \\
\varepsilon
\end{array}\right]=\underbrace{\left[\begin{array}{cc|c}
0 & I_{M} & I_{M} \\
I_{M} & 0 & 0 \\
\hline-I_{M} & I_{M} & I_{M}
\end{array}\right]}_{H_{2}}\left(\left[\begin{array}{c}
x \\
x^{p} \\
\hline n
\end{array}\right]\right),}
\end{gathered}
$$

with $x^{p}=P r^{p}, r^{p}=x$, and matrix, $H_{2}$, independent of uncertain interconnection matrix, $P$.

The Problem 1 is now solved by the following theorem according to a similar argument as in Section IV-B. 
Theorem 4 (Control Design): Given $\eta>0$ and $\gamma>0$, the system described in Eq. (17), and real scalars, $X \geq$ $0, Y, Z \leq 0$, if there exists positive-definite matrix, $Q \in \mathbb{R}^{M \times M}$ and a real scalar $D>0$, such that

(i) $\mathrm{H}_{2}$ is $\left\{\boldsymbol{d i a g}\left(X Q,-D,-\eta^{2} I\right)\right.$, diag $(Y Q, 0,0)$, diag $\left.\left(Z Q, \gamma^{2} D, I\right)\right\}$-dissipative,

and a local controller, $K$, such that for $T_{s}$ and $S$ in Eq. (5):

(ii) $T_{s}$ is strictly $\{-Z,-Y,-X\}$-dissipative with $\|\hat{T}(s)\|_{\infty}<1$, and $\hat{T}_{s}(s)=\left(T(s)+\frac{Y}{X}\right) \sqrt{\frac{X^{2}}{Y^{2}-X Z}}$;

(iii) $|S(j \omega)| \leq \omega \Omega_{K}^{-1}(\omega)$, in the $L F$ range;

(iv) $|K(j \omega) S(j \omega)| \leq \eta^{-1} \min \left\{\Omega_{K S}(\omega), \omega \Omega_{T}(\omega)\right\}$, in the HF range;

then the local controller $K$ solves the Problem 1 for all possible interconnection matrix $P$ satisfying

$$
\bar{\sigma}(P) \leq \gamma
$$

Proof: See [23] for the proof.

Remark 2: Conditions (ii)-(iv) of Theorem 3 are decentralized local conditions since they are only related to local subsystem dynamics. However, due to the condition $(i)$, these local conditions imply the appropriate global system behavior: global stability and global system performance defined in Problem 1. In the scalar case, it is possible to find $Q, \eta^{2}$ and $X, Y, Z$ dissipativity parameters (based on quasi-convex optimization) that, for a given $\gamma$, satisfy condition (i), and maximally relax condition (ii), see [21] for more details. These algorithms can be implemented locally at each sensor provided the information on the number of sensors, $M$, is available to each sensor. In this case, the controller design reduces to a local $H_{\infty}$ design problem, i.e., find a controller, $K$, such that it satisfies the conditions (ii)-(iv). It is a standard $H_{\infty}$ design problem and can be easily solved (see [27]) and implemented by each sensor for a computed $\eta$ and frequency constraints fixed by $\Omega_{K}, \Omega_{T}, \Omega_{K S}$.

\section{Simulations}

We consider a network of $M=20$ sensors and $N=m+1=3$ anchors in $\mathbb{R}^{2}$. The sensors lie in the convex hull (triangle) formed by the anchors The sensor deployment is assumed to be free as soon as each sensor is connected to three neighbors such that it lies in their convex hull and resulting network topology $P$ is such that it respects constraint (8).

First performance specification needed to be ensured by local controllers, $K$, is the sensor localization objective, $\lim _{t \rightarrow \infty} x=x^{*}$. Second is the rejection of the disturbance, $n$, each component of which is a realization of a bandlimited noise with amplitude, $A<5$, in frequency range, $\left[\omega_{z}^{-} \omega_{z}^{+}\right]=\left[\begin{array}{lll}600 & 10^{5}\end{array}\right] \mathrm{rad} / \mathrm{sec}$. To achieve the specifications, we consider the frequency constraints, $\Omega_{K}(\omega), \Omega_{T}(\omega), \Omega_{K S}(\omega)$, see Problem 1 and Table I, shown as red dotted lines in Fig. 2.

Based on the proposed approach, see Theorem 4, each sensor is able to compute its local controller, $K$, that ensures the global performance specifications. The only information needed to be transmitted to the sensors is the total number of sensors, $M=20$, in the network, the spectral bound for the network topology, $\gamma=0.99$, and frequency constraints $\Omega_{K}(\omega), \Omega_{T}(\omega), \Omega_{K S}(\omega)$, see Remark 2. With this information, the quasi-convex optimization problem proposed in [21] could be solved by each sensor, which for the global system description in Eq. (17), 
yields $X=-3.33, Y=1.17, Z=1$ and the global transfer function bound, $\eta=9.7$. The local controller, $K(s)$, is then computed using standard $H_{\infty}$-design, [27], to ensure the conditions (ii)-(iv) of Theorem 4:

$$
K(s)=\frac{8.8 \cdot 10^{8}}{(s+777.5)(s+7508)} \text {. }
$$

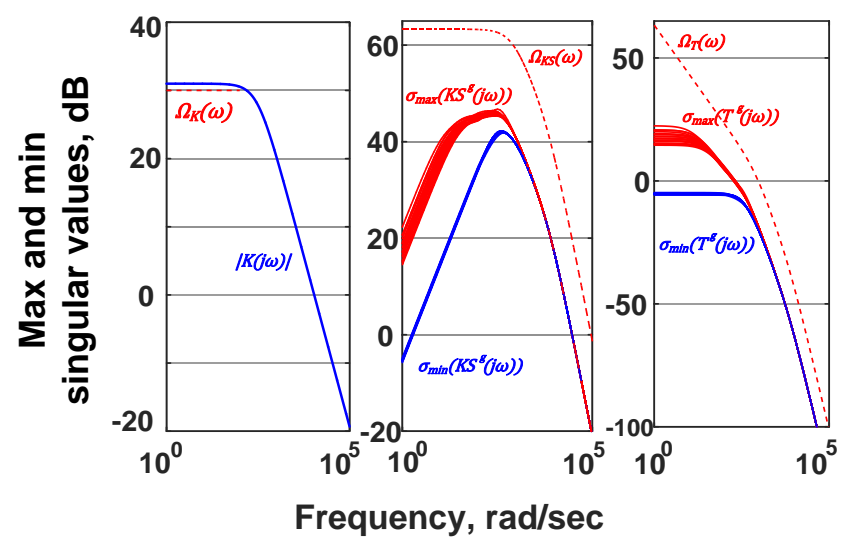

Fig. 2. Maximum (solid red line) and minimum (solid blue line) singular value of $K, K S^{g}$, and $T^{g}$ for different network topologies of 20 sensors, respecting constraint (8), together with corresponding frequency constraints (red dotted line).

As expected, the local controller is a low-pass filter with high gain, $K(0) \approx 150.9 \geq K_{0}$, in the Low Frequency (LF) range, and the negative slope $(-40 \mathrm{~dB} / \mathrm{dec})$ in the HF range. According to the Theorem 4 , the designed controller solves the Problem 1, i.e., it ensures the frequency constraints, Eq. (11), as can be verified in Fig. 2. It is important to note that even if the tracking performance specification was not directly imposed by the augmented global system under consideration, Eq.(17), since the global stability is ensured and the computed controller has no pure derivative actions, $K(0) \neq 0$, by Theorem 1 , the steady-state localization error $\varepsilon^{r e f}=x^{*}-x \rightarrow 0$. This is confirmed by temporal simulations of sensor network for 10 randomly chosen interconnection topologies that respect the constraint in Eq. (8). The temporal evolution of the mean value of localization errors, $\varepsilon_{i}^{r e f}$, and command signals, $y_{i}$ 's, are presented in Fig. 3. To illustrate the interest of using the dynamic controller instead of a static one, Fig. 3 presents also the temporal simulations for the case of proportional gain controller $K=\alpha=K_{0}$. Even though it ensures the same tracking performance (speed of convergence) as the dynamic controller proposed in this paper, it can be seen that due to the frequency constraint imposed on global transfer functions, $K S^{g}$ and $T^{g}$, the influence of the disturbance, $n$, at each sensor is significantly reduced.

\section{CONCLUSiOnS}

In this paper, we consider a continuous-time, LTI algorithm to solve the sensor localization problem in $\mathbb{R}^{m}$ with at least $m+1$ anchors which know their locations. Towards a more practical scenario, we consider the information exchange to incur a zero-mean additive disturbance whose frequency spectrum lies in a certain HF range. To maintain certain performance objectives while reducing the impact of the disturbance, we design a dynamic controller at 

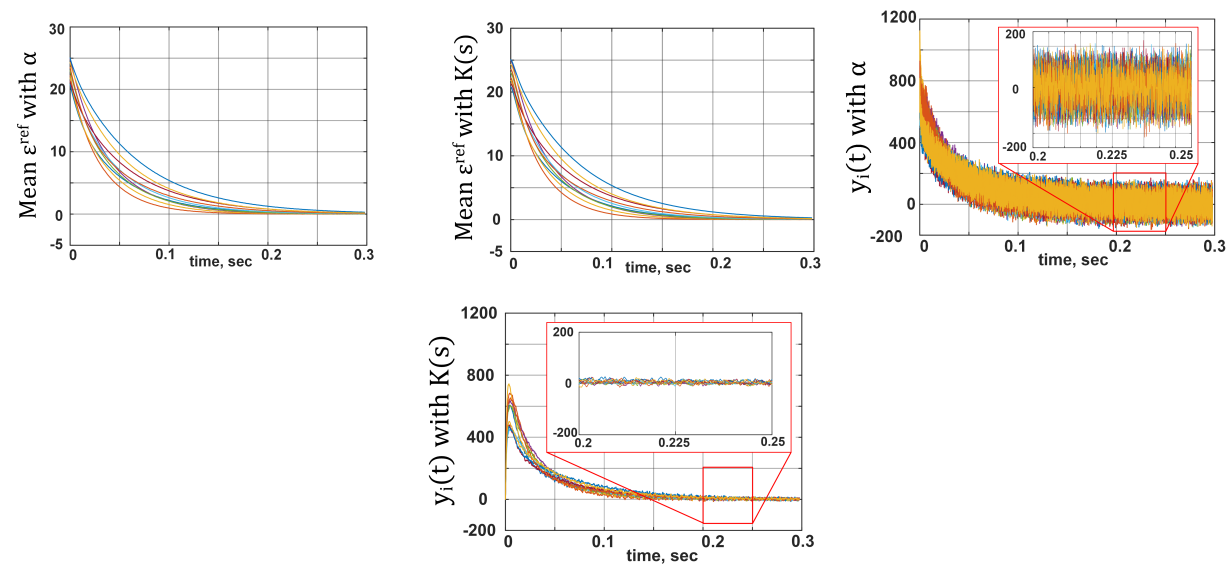

Fig. 3. Temporal simulations for 10 different topologies: (Left) Static controller, $\alpha=K_{0}$; (Right): Dynamic controller, $K(s)$. Note the high values of the control command, $y_{i}(t)$ 's, with the static controller, that could overexcite the local system, integrator, at each sensor.

each sensor with frequency-dependent performance objectives using the $H_{\infty}$ theory. This controller is computed with local information and relies on a spectral bound on the interconnection matrices. We show that this dynamic controller does not only ensure global system stability but is also able to meet certain performance objectives embedded in frequency-dependent constraints.

\section{REFERENCES}

[1] U. A. Khan, S. Kar, and J. M. F. Moura, "Linear theory for self-localization: Convexity, barycentric coordinates, and Cayley-Menger determinants," IEEE Access, vol. 3, pp. 1326-1339, 2015.

[2] R. L. Moses, D. Krishnamurthy, and R. Patterson, "A self-localization method for wireless sensor networks," EURASIP Journal on Applied Signal Processing, , no. 4, pp. 348-358, Mar. 2003.

[3] Y. Shang, W. Ruml, Y. Zhang, and M. Fromherz, "Localization from mere connectivity," in 4th ACM international symposium on mobile ad-hoc networking and computing, Annapolis, MD, Jun. 2003, pp. 201-212.

[4] Y. Shang and W. Ruml, "Improved MDS-based localization," in IEEE Infocom, Hong Kong, Mar. 2004, pp. 2640-2651.

[5] M. Cao, B. D. O. Anderson, and A. S. Morse, "Localization with imprecise distance information in sensor networks," in 44th IEEE Conference on Decision and Control, Sevilla, Spain, Dec. 2005, pp. 2829-2834.

[6] B. D. O. Anderson, I. Shames, G. Mao, and B. Fidan, "Formal theory of noisy sensor network localization," SIAM Journal of Discrete Mathematics, vol. 24, no. 2, pp. 684-698, 2010.

[7] G. Destino, Positioning in Wireless Networks: Non-cooperative and Cooperative Algorithms, Ph.D., University of Oulu, 2012.

[8] A. Savvides, H. Park, and M. B. Srivastava, "The bits and flops of the n-hop multilateration primitive for node localization problems," in Intl. Workshop on Sensor Networks and Applications, Atlanta, GA, Sep. 2002, pp. 112-121.

[9] R. Nagpal, H. Shrobe, and J. Bachrach, "Organizing a global coordinate system from local information on an ad-hoc sensor network," in 2nd Intl. Workshop on Information Processing in Sensor Networks, Palo Alto, CA, Apr. 2003, pp. 333-348.

[10] J. Albowicz, A. Chen, and L. Zhang, "Recursive position estimation in sensor networks," in IEEE Int. Conf. on Network Protocols, Riverside, CA, Nov. 2001, pp. 35-41.

[11] S. Čapkun, M. Hamdi, and J. P. Hubaux, "GPS-free positioning in mobile ad-hoc networks," in 34th IEEE Hawaii International Conference on System Sciences, Wailea Maui, HI, Jan. 2001, pp. 1-10.

[12] J. Fang, M. Cao, A. S. Morse, and B. D. O. Anderson, "Sequential localization of sensor networks," SIAM Journal on Control and Optimization, vol. 48, no. 1, pp. 321-350, 2009. 
[13] M. Deghat, I. Shames, B. D. O. Anderson, and J. M. F. Moura, "Distributed localization via barycentric coordinates: Finite-time convergence," in 18th IFAC World congress, Milano, Italy, Aug. 2011, pp. 7824-7829.

[14] A. T. Ihler, J. W. Fisher III, R. L. Moses, and A. S. Willsky, "Nonparametric belief propagation for self-calibration in sensor networks," in IEEE ICASSP, Montreal, Canada, May 2004.

[15] S. Thrun, "Probabilistic robotics," Communications of the ACM, vol. 45, no. 3, pp. 52-57, Mar. 2002.

[16] U. A. Khan, S. Kar, and J. M. F. Moura, "Distributed sensor localization in random environments using minimal number of anchor nodes," IEEE Transactions on Signal Processing, vol. 57, no. 5, pp. 2000-2016, May 2009.

[17] August Ferdinand Möbius, Der barycentrische calcul, 1827.

[18] U. A. Khan, A. Korniienko, and K. H. Johansson, "An $H_{\infty}$ - based approach for robust sensor localization," in Proc. IEEE Conf. on Decision and Control, Osaka, Dec. 2015, pp. 1719-1724.

[19] P. J. Moylan and D. J. Hill, "Stability criteria for large-scale systems," IEEE Trans. Aut. Control, vol. AC-23, no. 2, pp. 143-149, Apr. 1978.

[20] G. Scorletti and G. Duc, "An LMI approach to decentralized $H_{\infty}$ control," Int. J. Control, vol. 74, no. 3, pp. 211-224, 2001.

[21] A. Korniienko, G. Scorletti, E. Colinet, and E. Blanco, "Performance control for interconnection of identical systems: Application to PLL network design," International Journal of Robust and Nonlinear Control, vol. 26, no. 1, pp. 3-27, Jan. 2016.

[22] J. Doyle, A. Packard, and K. Zhou, "Review of LFT's, LMI's and $\mu$," in IEEE Conf. Decision and Control, IEEE, Ed., Brighton, England, Dec. 1991, vol. 2, pp. 1227-1232.

[23] A. Korniienko, U. A. Khan, and G. Scorletti, "Robust sensor localization with locally-computed, global $H_{\infty}$-design," Tech. Rep., Laboratoire Ampère, Ecole Centrale de Lyon, 2016.

[24] A. Korniienko, G. Scorletti, E. Colinet, E. Blanco, J. Juillard, and D. Galayko, "Control law synthesis for distributed multi-agent systems: Application to active clock distribution networks," in Proc. American Control Conf., San Francisco, June 2011, pp. 4691- 4696.

[25] V. A. Yakubovich, "The $\mathcal{S}$-procedure in non-linear control theory," Vestnik Leningrad Univ. Math., vol. 4, pp. 73-93, 1977, In Russian, 1971.

[26] A. Rantzer, "On the Kalman-Yakubovich-Popov lemma," Systems and Control Letters, vol. 27, no. 5, Jan. 1996.

[27] S. Skogestad and I. Postlethwaite, Multivariable Feedback Control, Analysis and Design, John Wiley and Sons Chischester, 2005. 